\title{
Agregar información sobre la duración de la jornada laboral mejora la predicción del riesgo cardiovascular
}

Using additional information on working ours helps on predicting cardiovascular risk

Kivimäki M, col. Ann Intern Med. 2011 Apr 5;154(7):457-63.

\section{Objetivos}

Examinar si el agregado de información sobre la duración de la jornada laboral mejora la capacidad del modelo de riesgo de Framingham para predecir enfermedad coronaria en una población de bajo riesgo cardiovascular laboralmente activa.

\section{Diseño}

Estudio prospectivo de cohortes.

\section{Lugar}

Oficinas estatales de Londres (estudio Whitehall II).

\section{Pacientes}

7.095 adultos (2.109 mujeres y 4.986 hombres) de 39 a 62 años de edad, con trabajo a tiempo completo, sin enfermedad coronaria al inicio del estudio. Se excluyeron aquellos con infarto de miocardio previo y con historial de angina identificados mediante cuestionario y confirmados por ECG en reposo, ECG de esfuerzo o angiografía coronaria.

\section{Evaluación de factores pronósticos}

Se midieron los factores de riesgo tradicionales (edad, sexo, hipertensión, dislipemia, diabetes, tabaquismo), y se clasificó a los pacientes de acuerdo a las recomendaciones actuales en bajo riesgo $(<5 \%)$, riesgo bajo a intermedio $(5 \mathrm{a}<10 \%)$ y riesgo intermedio a alto $(>10 \%)$. El resultado primario fue la aparición del primer infarto no fatal o muerte de causa coronaria.

\section{Resultados principales}

Ver tabla 1. Se registraron 192 eventos coronarios (29 muertes y 163 infartos no fatales) en los 12,3 años que duró el seguimiento. El riesgo relativo de los dos modelos de riesgo para la incidencia de un evento coronario en el grupo de mayor riesgo (>10\%) versus el de bajo riesgo $(<5 \%)$ fue de $3,91 \quad(95 \%$ IC 1,92 a 7,96$)$ y 5,39 (95\% IC 2,92 a 9,96$)$, respectivamente. El ajuste por jornadas laborales extensas (más de 11 horas) resultó en la reclasificación del riesgo en $4,7 \%$ de los pacientes (p 0,034).

Tabla 1: Riesgo** de sufrir un evento coronario según la duración de la jornada laboral.

\begin{tabular}{c|c}
$\begin{array}{c}\text { Duración de jornada } \\
\text { laboral }\end{array}$ & RR (IC95\%)*** \\
\hline $\mathbf{9}$ horas & $0,90(0,60$ a 1,35$)$ \\
\hline $\mathbf{1 0}$ horas & $1,45(0,99$ a 2,12$)$ \\
\hline $\mathbf{1 1}$ horas & $1,67(1,10$ a 2,55$)$ \\
\hline
\end{tabular}

** Ajustado por puntaje de Framingham. ${ }^{* * *}$ Comparado contra una jornada laboral de 7 a $8 \mathrm{~h}$.

\section{Conclusiones}

Incorporar información sobre la duración de la jornada laboral puede mejorar la performance del puntaje de Framingham para predecir eventos coronarios en trabajadores con bajo riesgo cardiovascular.

Fuente de financiamiento: Medical Research Council; British Heart Foundation; Wellcome Trust; National Heart, Lung, and Blood Institute; National Institute on Aging

\section{Comentario}

A principios del siglo pasado, Frederick Taylor revolucionó las relaciones de trabajo al demostrar que un régimen de ocho horas de trabajo y ocho horas de sueño diarias aumentaba la productividad y reducía los costos para el empleador ${ }^{1}$. Sus recomendaciones fueron adoptadas por empresarios como Henry Ford tanto como por los primeros sindicatos modernos. Este trabajo constituye un intento por mejorar la predicción de la mortalidad de causa coronaria mediante la incorporación de un factor de riesgo no tradicional (psicosocial) a una regla ya conocida como el score de Framingham. La relación entre jornadas extensas de trabajo y mortalidad de causa coronaria se conoce desde la década del ' $60^{2}$. Existen incluso estudios de cohortes y de casos y controles realizados en Europa, Estados Unidos y Asia que confirman esta relación, con significación estadística ${ }^{3,4,5}$. En el presente estudio, trabajar más de 11 horas se asoció una probabilidad $67 \%$ mayor de sufrir un evento coronario comparado con aquellos que trabajaban 7 a 8 horas, y el ajuste por jornadas laborales extensas efectivamente resultó en una mejor estimación del riesgo cardiovascular. Estos hallazgos, sin embargo, pertenecen a una población de bajo riesgo y no son extrapolables a otras pobla- ciones. Eventualmente esta propuesta debería investigarse en estas poblaciones y validarse en una cohorte independiente. Sin embargo, llama la atención que los autores concluyan que la información sobre la duración de la jornada laboral podría ayudar a predecir mejor el riesgo pero que "los beneficios clínicos de evitar las jornadas laborales extensas deberían ser demostrados".

\section{Conclusiones del comentador}

Compartir tiempo con la familia, disfrutar de una actividad física regular, descansar una cantidad adecuada de horas de sueño, forman parte de las recomendaciones que todos los médicos de familia del país comunicamos diariamente a nuestros pacientes, independientemente de la evaluación de su riesgo cardiovascular. Clínicamente es irrelevante incorporar información sobre la duración de la jornada laboral si no va a cambiar nuestra intervención al respecto, a menos que nos haga reflexionar a nosotros mismos sobre la pertinencia de seguir trabajando 24 horas continuas y más en las guardias.

Agustín González Calbano [ Unidad Sanitaria “Saladero” - Secretaría de Salud de Bahía Blanca drcalbano@yahoo.com.ar ]

González Calbano A. Agregar infomación sobre la duración de la jornada laboral mejora la predición del riesgo cardiovascular. Evid Act Pract Ambul. Ene-Mar 2012;15(1):13. Comentado de: Kivimäki M, Batty GD, Hamer M, y col. Using additional information on working hours to predict coronary heart disease: a cohort study. Ann Intern Med. 2011 Apr 5;154(7):457-63. PMID: 21464347.

\section{Referencias}

1. Taylor FW. The Principles of Scientific Management. New York, NY: Harper \& Brothers; 1911.

2 Buell P, Breslow L. Mortality from coronary heart disease in California men who work long hours. J Chronic Dis. 1960;11:615-26.

3. Theorell T, Rahe RH. Behavior and life satisfactions characteristics of Swedish subjects with myocardial infarction. J Chronic Dis. 1972;25:139-47.

4. Falger PR, Schouten EG. Exhaustion, psychological stressors in the work environment, and acute myocardial infarction in adult men. J Psychosom Res. 1992;36:777-86.

5. Sokejima S, Kagamimori S. Working hours as a risk factor for acute myocardial infarction in Japan: case-control study. BMJ. 1998;317:775-80. 